\title{
Femtosecond Laser Induced Nanowire Technique and Its Applications
}

\author{
Motoyoshi Baba, ${ }^{1,2}$ Tianqing Jia, ${ }^{3}$ Masayuki Suzuki, ${ }^{4}$ and Hiroto Kuroda ${ }^{2}$ \\ ${ }^{1}$ Institute for Solid State Physics, University of Tokyo, Chiba 277-8581, Japan \\ ${ }^{2}$ Department of Ophthalmology and Advanced Laser Medical Center, Faculty of Medicine, Saitama Medical University, \\ Saitama 350-0495, Japan \\ ${ }^{3}$ State Key Laboratory of Precision Spectroscopy, East China Normal University, Shanghai 200062, China \\ ${ }^{4}$ Kansai Photon Science Institute, Japan Atomic Energy Agency, Kyoto 619-0215, Japan
}

Correspondence should be addressed to Motoyoshi Baba, baba@issp.u-tokyo.ac.jp

Received 17 March 2011; Accepted 18 April 2011

Academic Editor: A. W. Mohammad

Copyright (C) 2011 Motoyoshi Baba et al. This is an open access article distributed under the Creative Commons Attribution License, which permits unrestricted use, distribution, and reproduction in any medium, provided the original work is properly cited.

\begin{abstract}
Semiconductor nanowires are very attractive due to their interesting fundamental properties and enormous potentials for device applications to the nanoscale optoelectronics and solar cells, and so forth. We fabricated semiconductor nanowires of various wire parameters such as wire length, diameter, and density by femtosecond laser induced method. We report the development of our technology of creating semiconductor nanowires with smaller size than the laser wavelength at precise position by femtosecond laser ablation technique. There are a variety of growth methods for nanowires including chemical vapor deposition (CVD), molecular-beam epitaxy (MBE), and pulsed laser deposition (PLD). Although PLD has recently been applied as a growth method for nanowires, laser induced nanowires are very good in quality. Their growth rate is much higher than that of nanowires grown by other ways. We discuss the growth mechanism of laser induced nanowires and describe the advantages of this approach.
\end{abstract}

\section{Introduction}

There are so many attracting nanostructure devices such as quantum well structures, and quantum wire structures, quantum dots. Especially semiconductors and silicon nanowires attract much attention because of their very interesting physical properties [1] and applications to solar cells [2]. This fact is industrially very important. Many studies on nanowire synthesis have been done $[3,4]$ and nanowire structures have been actively applied for research devices in many fields. Many researchers and technical experts in the world are competing fiercely for developing manufacturing technology of nanometer-scale materials. There are several types of nanowires such as metal nanowires (gold, copper, nickel, cobalt tin, etc.), semiconductor nanowires (cadmium sulfide, cadmium selenide, zinc selenide, etc.), and polymer nanowires (carbon nanotubes, polymers, etc.). These nanowires can be applied for many devices such as high-density and high-efficiency energy-conversion devices (solar cells/thermocouple), high-density data storage (phasechange random access memory), high sensitivity sensors (chemical sensors, bio sensors), high-speed/large-capacity optical connectors (light-emitting diodes/laser, photodetectors).

More specifically, silicon material is used for electronics and sensors, germanium for electronics and IR detectors, tin oxide for chemical sensors, indium oxide for chemical sensors and biosensors, indium tin oxide for transparent conductive films in display electrodes, solar cells, and organic light-emitting diodes, zinc oxide for ultraviolet UV laser, field emission device, and chemical sensors, copper oxide for field emission devices, gallium nitride for high-temperature electronics, UV detectors, lasers, automotive electronics, and sensors, boron nitride for insulators, indium phosphate for electronics and optoelectronics, zinc selenide for photonics (Q-switch, blue-green laser diode, blue-UV photodetector), copper and tungsten for electrical interconnects, and so 
forth. Because an interesting physical property of a nanostructure emerges when the structure size becomes nanosized, ultrafine processing technology is an important problem. In fact, diameters of carbon nanotubes are about several $\mathrm{nm}$, and they show good electrical conductivity; thus, they are expected as a wiring material for future electronic devices and have been studied so energetically. For nanodevice fabrication, there are typically two fabrication methods. One method is the so-called bottom-up technique, that is, the method of the miniaturization of semiconductor material, and the other is the bottom-up technique, that is, the method of assembling devices from atoms and molecules. Electrically conductive metal nanowires (diameter of nanometer-scale metallic wires) are expected to be used for wiring material as with carbon nanotubes. Photolithography processing is in the mainstream of the current wiring manufacturing, but machining accuracy is limited due to the wavelength of light. Thus several hundred $\mathrm{nm}$ or less machining is difficult in this way. For good electrical conductive metal nanowires such as gold, silver, copper and other metals, there have been many reports of various ways of synthesization for something close to a small needle-like crystals. The length of nanowires synthesized in any way is only about thousands of $\mathrm{nm}$, that is the reason they did not become useful material.

Thinning technology for electrically conductive nanowire is one that is an integral part of the process technology needed to produce the next generation of integrated circuits. Because nanowire structure is one-dimensional geometry, there are two quantum wire structure types. One structure type is the type where the wire axis is parallel to the substrate and the other type is perpendicular to the substrate. To fabricate wire structure perpendicular to the substrate, the microfabrication etching technology has been used.

Thus the size of the wire diameter is determined by the precision of the performance of fabrication technology. We report the development of our technology to create semiconductor nanowires with more accuracy than the wavelength by laser ablation technique.

\section{Nanowires Fabricated by Femtosecond Laser Ablation Method}

For making ultraprecise and ultra-high-density semiconductor devices, electronic devices need to be integrated in nanoscale. The nanometer-scale ultrafine processing has become essential. Developing of semiconductor nanowire technology and its application to electronic devices allows further evolution of electronics technology beyond the limits of silicon LSI semiconductor technologies.

Photolithography is currently the main stream method of processing wire. But the processing of one hundred nanometers or less is difficult in this method. Various research and development have been done for the development of wiring materials to electronic devices.

Semiconductor nanowires are the structures which are "one-dimensional semiconductor structures with a diameter of nanoscale." Low dimensionality of materials causes low consumption of energy, high efficiency, and super integration of semiconductor circuits. New features in large engineering applications will be expected.

Semiconductor nanowire has a single-crystal structure and its electrical property varies greatly depending on doping materials. Thus, the fabrication method of selectively forming compound semiconductor nanowires, integrated structure, and exploration of properties become extremely important factors for nanowire application and devices for the development of new functional devices and circuits. Nanowire structures, as tiny integrated circuits or the wiring material for quantum devices, are expected to be applied as an electron emission source, such as flat panel and molecular devices.

Nanowires can be synthesized by the bottom-up method such as grown metalorganic vapor phase epitaxy (SAMOVPE) and selective area metalorganic vapor phase epitaxy (SA-MOVPE). Key parameters such as chemical composition, length, diameter, doping, and so forth can be controlled during synthesis.

For example, an average diameter of created InP nanowire crystals is about $20 \mathrm{~nm}$, and reproducibility of formed nanowire crystals with a uniform diameter is very good. The wavelength of the emission characteristic has become shorter than that of the emission characteristics of the bulk one at room temperature. This fact agrees well with results from quantum confinement effects of carriers bound in the direction of the wire diameter. In this paper, we report the so-called top-down processing technology to introduce miniaturization of semiconductor material that we developed.

Nanostructures created by laser ablation method have been reported so far. Their periodicity is about half of the wavelength of the laser beam and is explained by the interference effect [5-8].

Semiconductor nanowires are very attractive due to their interesting fundamental properties and enormous potentials for device applications to the nanoscale optoelectronics and solar cells, and so forth. There are a variety of growth methods for nanowires which can control the growth orientation and cross-section such as chemical vapor deposition (CVD) $[9,10]$, molecular-beam epitaxy [11], and pulsed laser deposition (PLD) $[12,13]$. Among these methods, PLD has recently been applied to grow nanowires with high quality. We obtained very uniform semiconductor nanowires with smaller size than the laser wavelength at precise position by the new femtosecond laser ablation technique [14-16]. We controlled the nanowire length, diameter, and density by adjusting the laser parameters such as pulse width, pulse energy, pulse intensity, and repetition rates. We irradiated the semiconductor sample with a commercial titanium sapphire regenerative amplifier and an oscillator system (Tsunami: Spectra-Physics). The p-polarized pulse width is about 130 femtoseconds of $0.7 \mathrm{~mJ}$ at $1 \mathrm{kHz}$ repetition rate at $800 \mathrm{~nm}$ wavelength. We can control the laser energy continuously using a Glan polarizer and also adjust a laser intensity and pulse width. The laser beam was focused through a silica lens $(L=150-250 \mathrm{~mm})$ and irradiated vertically down to the upper surface of a zinc selenide wafer. 
The obtained crystalline nanowires were very uniform in diameter. The time of irradiation is short enough to melt the substrate materials, so this uniformity was attributed to the nonthermal ablation of the precursor materials by the femtosecond laser, which can induce the formation of uniform nanostructures. The zinc selenide wafer is undoped single crystal with orientation of 111, and both of its surfaces are polished, with roughness less than $15 \mathrm{~nm}$. After irradiation, we rinsed the sample elaborately with acetone, ethanol, and deionized water. We measured the sample with scanning electronic microscope (SEM:JEOL/JSM-5600), energy-dispersive X-ray spectroscopy (EDXS), micro-Raman spectroscopy (MRS), and a transmission electron microscope (TEM: JEOL/JEM-2010F). We show SEM images of the nanowire made by this method in Figure 1. Nanoripples are formed on the laser focusing point. Surprisingly, most nanowires grow on the nanoripples in the craters, while the neighboring area is unaffected. The SEM image in Figure 1(a) indicates that zinc selenide nanowires grow toward the focusing center. Therefore, we can focus the laser beam to the designed point and easily grow nanowires without polluting other parts of the sample surface. The needle-like nanowires were effectively fabricated in high density. Their lengths are about $1.3 \mu \mathrm{m}$ long (Figure 1(b)), with smooth sidewalls. The diameter at the nanowire root is about $80 \mathrm{~nm}$ and only $30 \mathrm{~nm}$ at its top. The SEM image also shows quasialigned nanowire arrays grown on the sidewall of the ablation craters (see Figure 2). The ZnSe nanowires were scraped off with a stainless steel needle, and their structure analyzed by TEM. It is about $120 \mathrm{~nm}$ in diameter with a smooth sidewall. Figure 3(a) shows the high-resolution TEM (HRTEM) image and the Fourier transform of the square part, respectively. The lattice space indicates that the nanowires are wurtzite single crystal growing along the $a$-axis. The $\mathrm{ZnSe}$ nanowires reported to date usually have a zinc blende structure growing along the (111) direction [17]. Wurtzite ZnSe nanowires growing along the $c$-axis were recently fabricated with PLD and thermochemical methods $[18,19]$. Nanowires with a wurtzite structure can also grow rapidly along the $a$-axis. $\mathrm{ZnO}$ is very similar to $\mathrm{ZnSe}$, which is also a II-VI group semiconductor with wurtzite structure and wide band gap $(3.1 \mathrm{eV})$.

The pulse width was short enough to melt the semiconductor surface, so the ablated plume expands and interacts with the air around and cools down rapidly. A lot of nanoclusters and nanoparticles deposit on the sample surface [20]. The boiling point of Se (957 K) is lower than that of $\mathrm{Zn}$ $(1180 \mathrm{~K})$, and it easily vaporizes. An energy-dispersive X-ray spectrograph (EDXS) analyzes the chemical composition of the nanowire. Figure 3(b) shows an EDXS of the nanowires obtained in this study. That image shows no contaminations except specific copper line originating from sample holder and fabricated nanowires which are very pure zinc selenide crystal. The $\mathrm{Zn}$ :Se molar ratio in the middle and the top part of nanowire is $1: 1$, which is the same as that of ZnSe crystal. However, the ratio is $58: 42$ at the nanowire root. This proposes a vapor-liquid-solid mechanism for the formation of $\mathrm{ZnSe}$ nanowires. During the femtosecond laser irradiation, dense plasma is formed in the sample surface

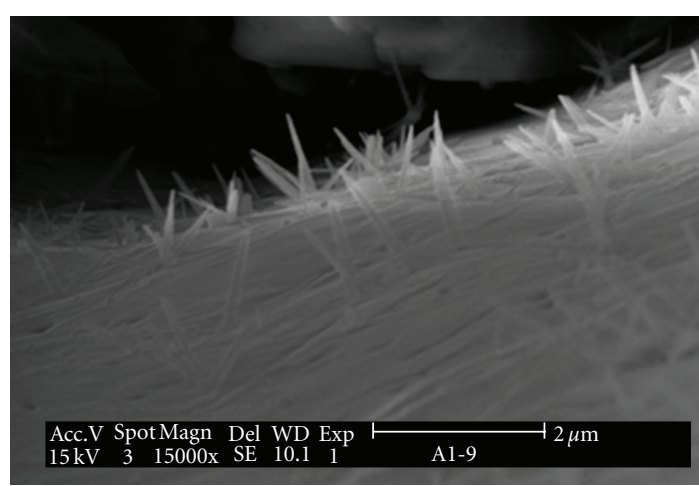

(a)

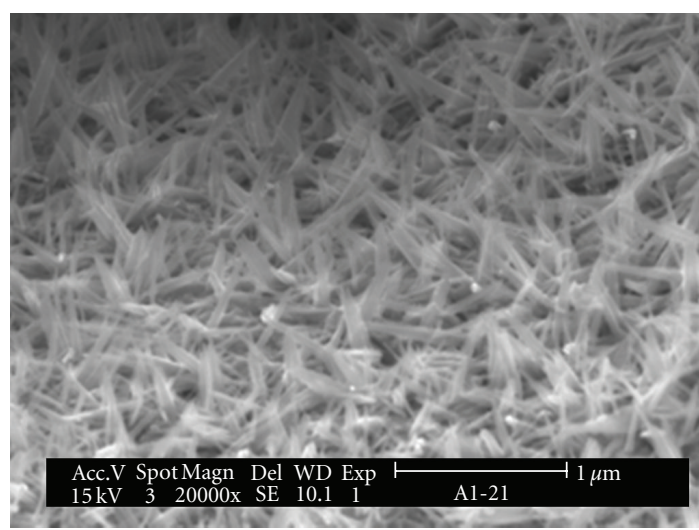

(b)

FIGURE 1: SEM images of the femtosecond laser induced nanowires. The linear polarized laser beam of $1 \mathrm{~mW}$ power irradiated the surface of zinc selenide substrate 500 times. (a) The spot size is about $3 \mathrm{~mm}$, and craters were made in the center of the laser beam. (b) The length of grown nanowires is about an order of $1 \mu \mathrm{m}$.

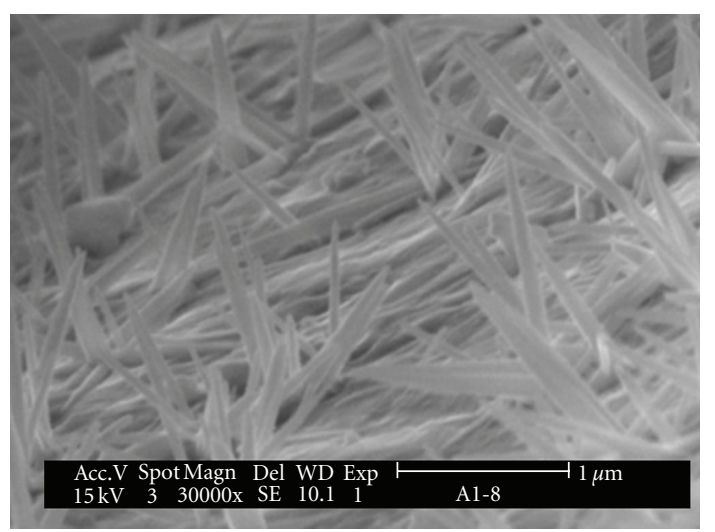

FIGURE 2: SEM images of the quasialigned nanowires irradiated by linear polarized $60 \mathrm{~mW}$ laser at $800 \mathrm{~nm}$ wavelength.

via two-photon absorption and impact ionization, which will further intensively absorb laser energy. The energy density deposited in the sample surface is much higher than $10 \mathrm{~kJ} / \mathrm{cm}^{3}$ [21], which induces ZnSe crystal to vaporize and decompose into $\mathrm{Zn}$ and Se. Therefore, the molar ratio of $\mathrm{Zn}:$ Se in these nanoparticles is larger than $1: 1$. 


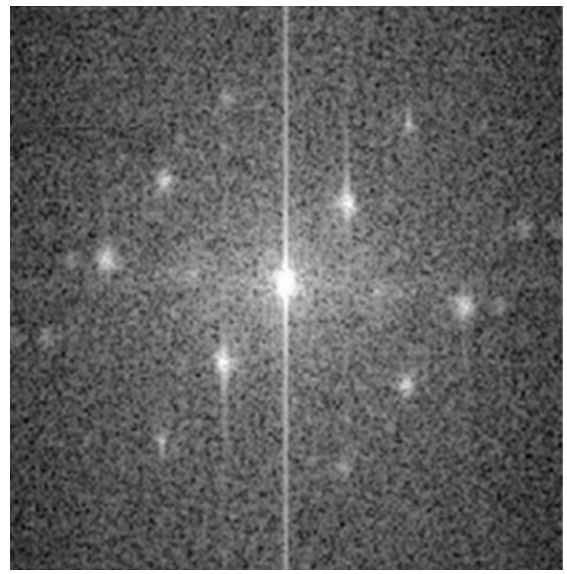

(a)

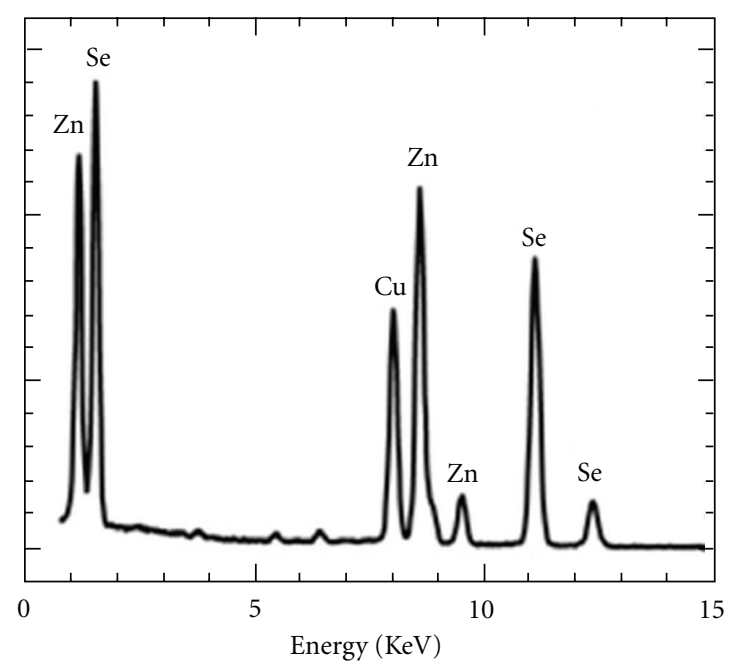

(b)

Figure 3: (a) The HRTEM image and Fourier transform of the square part; (b) EDXS spectrum of ZnSe nanowire.

Figure 4 shows the growth process of the nanowires. Nanowires begin to nucleate and grow after the sample is irradiated by 100 laser pulses (Figure 4(a)) and by 500 laser pulses (Figure $4(\mathrm{~b})$ ). Their lengths increase linearly to $1.7 \mu \mathrm{m}$ as the pulse number increases to 1500 (Figure 4(c)). The growth rate is $1.2 \mathrm{~nm} /$ pulse. We found the very rapid nanowire growth rate of $2-10 \mu \mathrm{m} / \mathrm{s}$. This growth rate is much higher than that of CVD method by a factor of $10^{3}-10^{4}$ (the latter is usually $0.5-3 \mathrm{~nm} / \mathrm{s}$ ) [17]. However, if the pulse number increases further, the length of the nanowires begins to decrease slowly (Figure $4(\mathrm{~d})$ ). This is because the laser ablation of the ZnSe crystal surface and the deposited nanoclusters induce the growth of nanowires, while the laser can also ablate the nanowires and cut down their lengths. Micro-Raman spectroscopic (MRS) analysis is also performed on the nanowires. Raman spectral peaks at 251 and $205 \mathrm{~cm}^{-1}$ are attributed to the longitudinal and the transverse optical modes. Compared with those of $\mathrm{ZnSe}$ crystals, both of the two peaks are wider because the surface-volume ratio of nanowires is much higher [18]. No vibration mode of $\mathrm{ZnO}$ is observed; hence, we confirmed that evaporated zinc atoms are not oxidized and no $\mathrm{ZnO}$ molecules are formed during femtosecond laser ablation, though it is conducted in air. Thus the formation mechanism is studied and found to be self-catalyzed vapor-liquid-solid process. We also formed aligned nanoparticles on the surface of $6 \mathrm{H}-\mathrm{SiC}$ fabricated by two collinear femtosecond laser beams $[6,22]$.

Our discovery opens a way to rapidly and simply grow nanowires at the designed positions in microscopic optoelectronics and other device applications. Due to specific adsorption of polar molecules by surface-specific and selective detection, some zinc compounds can be applied highly sensitive detectors of pharmacological device sensors (DNA sensor, sensor to determine the efficacy). Zinc oxide nanorods have also been applied for gate-type FETs. Using laser induced VLS (vapor-liquid-solid) method, under the optimized conditions of temperature and catalyst size, highly oriented zinc oxide nanorods of large surface are formed [23].

\section{Various Applications such as Solar Cells}

The high intensity of femtosecond laser optical fields can inject the light energy inside the material efficiently before thermal equilibrium of lattice electrons; thus photo-induced electron plasma wave (plasmon) and laser pulse (photons) can interact. In particular, in case of silicon oxide $\left(\mathrm{SiO}_{2}\right)$, the nano-grating structure is formed due to oxygen vacancies, that is, the composition rate of oxide varies, and the appearance of $\mathrm{SiO}_{1.4}$ structure has been reported [24]. Nano-grating structure formation is derived from the plasma induced by laser light and has been found to be particularly dependent on the plasma electron temperature. These nanostructures such as nanogratings, polarization separator, and microoptical attenuators can be integrated inside the glass or fibers and will be applied to the ultracompact optical devices. We can use these materials with periodic microstructures as a substrate for the growth of single-crystal substrate in this way, and also we will be able to create quantum device elements on it. In addition to optoelectronic devices, the silicon nanowires are expected to be applied to solar cells and are the most important candidates for next-generation solar cell technology. Energy from the sun pours down on earth at the ratio of two calories per second per unit surface area in fine weather, and the energy is equivalent to the output power of about 1 kilowatt per square meter. In other words, the sun radiates more than a huge 127 billion kwh energy per hour from 150 million $\mathrm{km}$ beyond the earth. Considering that annual energy consumption of the world is equivalent to approximately 80 million tons of oil which is about 93 billion kwh electricity power conversion, how enormous the radiation energy from the sun on earth is. 


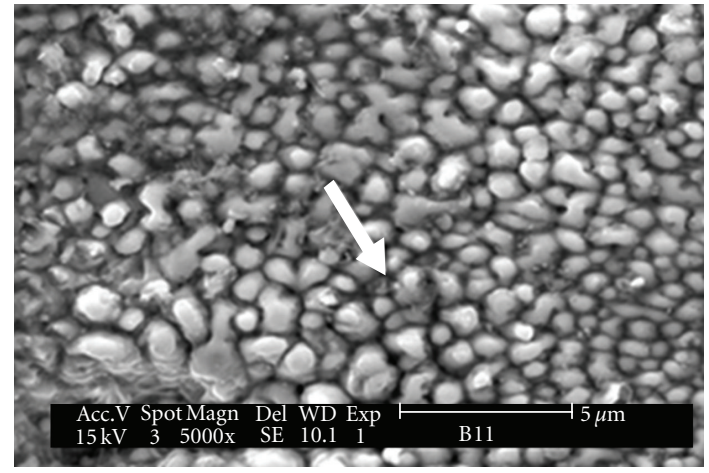

(a)

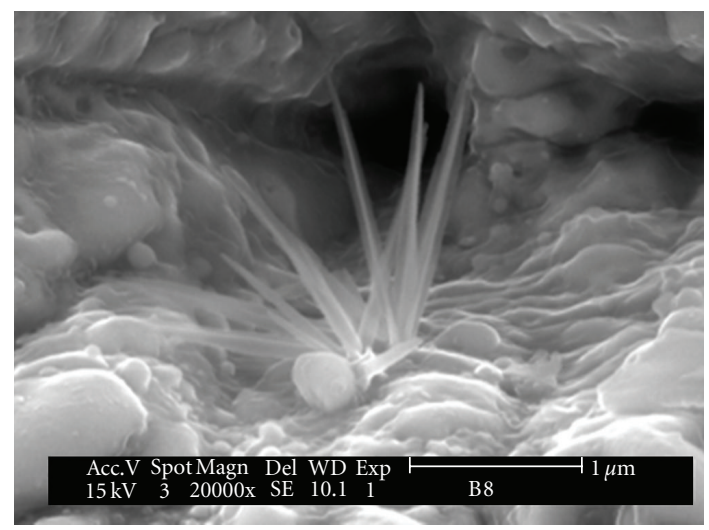

(c)

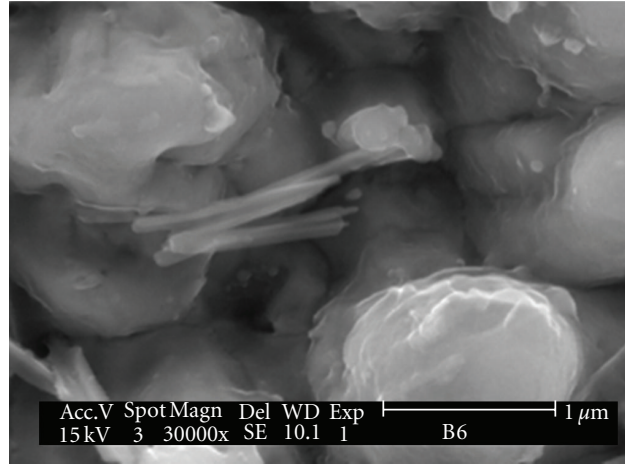

(b)

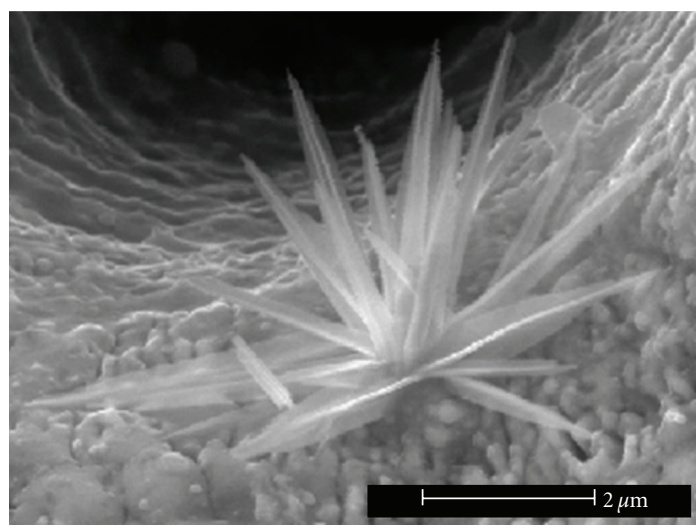

(d)

FIGURE 4: SEM images of nanowires. The arrow shown in (a) was the seed of nanowire. The number of laser shots were 100 times for (a), 500 times for (b), 1500 times for (c), and 2500 times for (d). The laser power was $18 \mu \mathrm{J}$ at $800 \mathrm{~nm}$ wavelength.

And more advantageous point is that solar energy is carbon dioxide gas free and a totally clean energy. Therefore the most important point for developing solar cells is how to reduce cost for making highly efficient solar cells. Under the concept of this largeness and cleanliness of solar energy, the tailwind blows to the development of solar energy, which foresees the future "age of the sun." In addition to the extrication from dependence on oil, a sense of prevention of global warming assists this trend of energy saving. In this trend, photovoltaic solar cells will shoulder a large amount of solar power, and evolve technically, and emerge as next-generation solar cells $[3,25,26]$. In case of silicon material, theoretical limit of conversion efficiency for even more powerful singlecrystal solar cell as compared to polycrystalline ones is to the utmost about 25-30\%. In the case of the amorphous solar cell, it does not cost in fabrication so much as compared to crystalline one, so amorphous solar cells are in front of a practical application. Nevertheless the theoretical limit of conversion efficiency is as low as $15 \%$. In the case of silicon single crystal-type ones, the product of the university of New South Wales in Australia recorded $24.4 \%$ of maximum efficiency. And they also recorded $19.8 \%$ of maximum efficiency in the case of polycrystalline type ones. The efficiencies of commercial ones for electric power generation supplied by Sharp Corporation or SANYO Electric Co., Ltd are around $12-16 \%$. Regardless of which method adopted, there is a limit to the conversion efficiency, and we cannot overcome the disadvantage of using large amount of silicon for solar cell. However the high quantum efficiency of solar cells using silicon nanowires is said to be achieved 30\% or above, so we can save the amount of silicon usage. For the next-generation solar cells, the technique of accumulating nanowires on large area must be developed. Many ideas for realization have been submitted. One idea is that to arrange thin wire-like silicon (silicon nanowire) on the substrate. This idea is already realized around the world. For example, the method having been realized by General Electric Company in USA is to reduce the amount of silicon by adopting the wire-like shape of silicon, which results in low cost. And they aim to get higher efficiency with reducing the reflection of light by overgrown nanowires. IMEC (Interuniversity Microelectronics Centre-an international research organization headquartered in the city of Leuven, Belgium) tries to fabricate silicon nanowires suitable for solar cells. IMEC aims to produce new type of silicon solar cells as forming nanowires on silicon substrates. Whereas the band gap of silicon substrate is $1.1 \mathrm{eV}$, the band gap of silicon nanowire becomes $1.7 \sim 1.8 \mathrm{eV}$ by utilizing quantum mechanical effects. The combination of different band gaps such as silicon substrate and silicon nanowire will enable to realize high efficiency. In this case, conversion efficiency would become about $33 \%$. If we attain the $1.7 \sim 1.8 \mathrm{eV}$ band 
gap, silicon nanowire diameter of $2 \sim 4 \mathrm{~nm}$ are needed. To form such very thin silicon nanowires, IMEC utilized EUV lithography for next-generation semiconductor manufacturing. Many studies have been advanced in the world in this way.

\section{Conclusion}

Thus nanowires are extremely interesting in terms of not only physical properties but device applications. They are a strategically important tool for energy-saving technology in the future. There is no established method for producing that can control various parameters. If we are to be able to produce large quantities quickly and efficiently at low cost, we can contribute significantly to the ubiquitous society. In addition to the understanding fundamental properties, it is highly desirable that the development of production method should be promoted energetically. We have developed laser ablation method for fabricating semiconductor nanowires, without any furnace, vacuum chamber, or additional metal catalyst, which can very efficiently make nanowires at precise position. The rate of growth of the nanowire irradiated by a fs laser is $1-3 \mu \mathrm{m} / \mathrm{s}$, and this value is higher than that of the CVD method by a factor of $10^{3}$. Our discovery opens a way to grow nanowires rapidly and simply on a designed micro pattern. Further development of this high-potential method is expected to create devices for application.

\section{Acknowledgment}

This work was partly supported by the Japan Society for the Promotion of Science (JSPS Fellowship no. P06045) and the Grant-in-Aid for Creative Scientific Research (14GS0206) of the Japan Society for the Promotion of Science (JSPS).

\section{References}

[1] S. Bhunia, T. Kawamura, Y. Watanabe, S. Fujikawa, and K. Tokushima, "Metalorganic vapor-phase epitaxial growth and characterization of vertical InP nanowires," Applied Physics Letters, vol. 83, no. 16, pp. 3371-3373, 2003.

[2] L. Tsakalakos, J. Balch, J. Fronheiser, B. A. Korevaar, O. Sulima, and J. Rand, "Silicon nanowire solar cells," Applied Physics Letters, vol. 91, no. 23, Article ID 233117, 2007.

[3] S. Kodambaka, J. Tersoff, M. C. Reuter, and F. M. Ross, "Diameter-independent kinetics in the vapor-liquid-solid growth of Si nanowires," Physical Review Letters, vol. 96, no. 9, Article ID 096105, 4 pages, 2006.

[4] A. Jamshidi, P. J. Pauzauskie, P. J. Schuck et al., "Dynamic manipulation and separation of individual semiconducting and metallic nanowires," Nature Photonics, vol. 2, no. 2, pp. 86-89, 2008.

[5] S. Kanehira, J. Si, J. Qiu, K. Fujita, and K. Hirao, "Periodic nanovoid structures via femtosecond laser irradiation," Nano Letters, vol. 5, no. 8, pp. 1591-1595, 2005.

[6] T. Q. Jia, F. L. Zhao, M. Huang et al., "Alignment of nanoparticles formed on the surface of $6 \mathrm{H}-\mathrm{SiC}$ crystals irradiated by two collinear femtosecond laser beams," Applied Physics Letters, vol. 88, no. 11, Article ID 111117, 3 pages, 2006.
[7] M. Y. Shen, C. H. Crouch, J. E. Carey, and E. Mazur, "Femtosecond laser-induced formation of submicrometer spikes on silicon in water," Applied Physics Letters, vol. 85, no. 23, pp. 5694-5696, 2004.

[8] Y. Shimotsuma, P. G. Kazansky, J. Qiu, and K. Hirao, "Selforganized nanogratings in glass irradiated by ultrashort light pulses," Physical Review Letters, vol. 91, no. 24, Article ID 247405, 4 pages, 2003.

[9] T. Kuykendall, P. J. Pauzauskie, Y. Zhang et al., "Crystallographic alignment of high-density gallium nitride nanowire arrays," Nature Materials, vol. 3, no. 8, pp. 524-528, 2004.

[10] J. B. Hannon, S. Kodambaka, F. M. Ross, and R. M. Tromp, "The influence of the surface migration of gold on the growth of silicon nanowires," Nature, vol. 440, no. 7080, pp. 69-71, 2006.

[11] Y. Cai, S. K. Chan, I. K. Sou, Y. F. Chan, D. S. Su, and N. Wang, "The size-dependent growth direction of $\mathrm{ZnSe}$ nanowires," Advanced Materials, vol. 18, no. 1, pp. 109-114, 2006.

[12] A. M. Morales and C. M. Lieber, "A laser ablation method for the synthesis of crystalline semiconductor nanowires," Science, vol. 279, no. 5348, pp. 208-211, 1998.

[13] Y. Zhang, R. E. Russo, and S. S. Mao, "Femtosecond laser assisted growth of $\mathrm{ZnO}$ nanowires," Applied Physics Letters, vol. 87, no. 13, Article ID 133115, 3 pages, 2005.

[14] T. Q. Jia, H. X. Chen, M. Huang et al., "ZnSe nanowires grown on the crystal surface by femtosecond laser ablation in air," Applied Physics Letters, vol. 89, no. 10, Article ID 101116, 2006.

[15] T. Jia, M. Baba, M. Huang et al., "Femtosecond laser-induced $\mathrm{ZnSe}$ nanowires on the surface of a $\mathrm{ZnSe}$ wafer in water," Solid State Communications, vol. 141, no. 11, pp. 635-638, 2007.

[16] T. Jia, M. Baba, M. Suzuki et al., "Fabrication of twodimensional periodic nanostructures by two-beam interference of femtosecond pulses," Optics Express, vol. 16, no. 3, pp. 1874-1878, 2008.

[17] Y. Li, Y. Bando, and D. Golberg, "Single-crystalline $\alpha$-Al2O 3 nanotubes converted from Al4O $4 \mathrm{C}$ nanowires," Advanced Materials, vol. 17, no. 11, pp. 1401-1405, 2005.

[18] Y. Jiang, X. M. Meng, W. C. Yiu et al., "Zinc selenide nanoribbons and nanowires," Journal of Physical Chemistry B, vol. 108, no. 9, pp. 2784-2787, 2004.

[19] Y. C. Zhu and Y. Bando, "Preparation and photoluminescence of single-crystal zinc selenide nanowires," Chemical Physics Letters, vol. 377, no. 3-4, pp. 367-370, 2003.

[20] S. J. Henley, G. M. Fuge, and M. N. R. Ashfold, "Comparing the short and ultrashort pulsed laser ablation of LiF," Journal of Applied Physics, vol. 97, no. 2, Article ID 023304, 9 pages, 2005.

[21] A. Ben-Yakar and R. L. Byer, "Femtosecond laser ablation properties of borosilicate glass," Journal of Applied Physics, vol. 96, no. 9, pp. 5316-5323, 2004.

[22] X. Jia, T. Q. Jia, L. E. Ding et al., "Complex periodic micro/ nanostructures on $6 \mathrm{H}-\mathrm{SiC}$ crystal induced by the interference of three femtosecond laser beams," Optics Letters, vol. 34, no. 6, pp. 788-790, 2009.

[23] T. K. Saito, M. Seki, and H. Tabata, "Self-organized ZnO nanorod with photooxidative cell membrane perforation enables large-scale cell manipulation," Analytical and Bioanalytical Chemistry, vol. 391, no. 7, pp. 2513-2519, 2008.

[24] N. Fukata, T. Oshima, N. Okada et al., "Phonon confinement in silicon nanowires synthesized by laser ablation method," Physica B, vol. 376-377, pp. 864-867, 2006. 
[25] D. Li, Y. Wu, R. Fan, P. Yang, and A. Majumdar, "Thermal conductivity of Si/SiGe superlattice nanowires," Applied Physics Letters, vol. 83, no. 15, pp. 3186-3188, 2003.

[26] A. I. Hochbaum, R. Chen, R. D. Delgado et al., "Enhanced thermoelectric performance of rough silicon nanowires," Nature, vol. 451, no. 7175, pp. 163-167, 2008. 

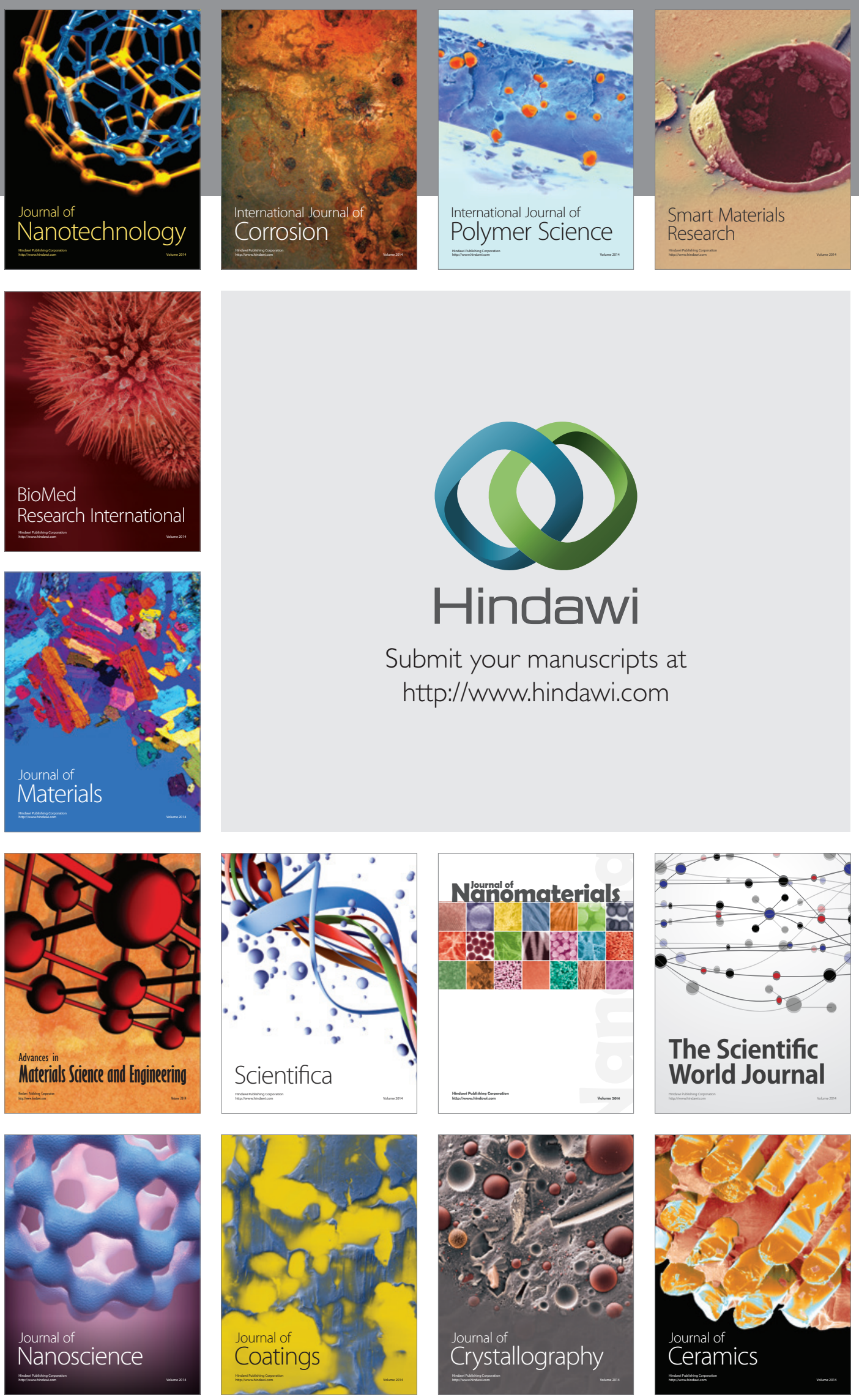

The Scientific World Journal

Submit your manuscripts at

http://www.hindawi.com

\section{World Journal}

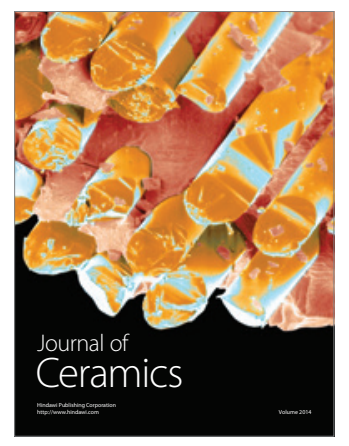

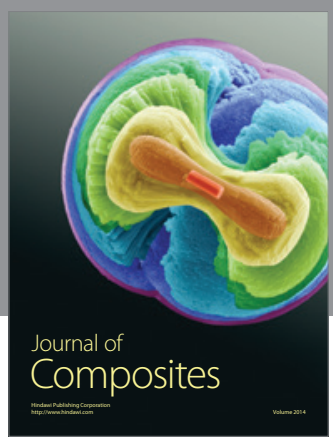
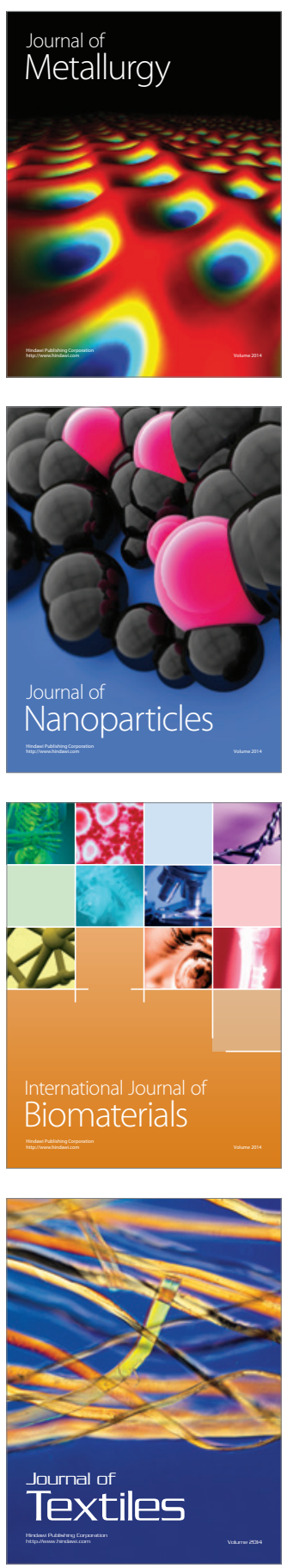